\title{
Reduction and Refinement Choices
}

Brandon Mercado (Brandon.Mercado@yale.edu)

Patrick Holland (patrick.holland@yale.edu)

Ilija Coric (ilija.coric@chem.uzh.ch)

Data reduction, modeling choices, and checkClF alerts are some of the last hurdles to consider before finalizing diffraction data before publication. My presentation will focus on organometallic structures with disorder and ambiguous space groups. Some of the reduction and refinement choices I made generated checkCIF alerts that required some consideration and explanations. 